Pacific

Journal of

Mathematics

\title{
DIFFERENTIAL HARNACK ESTIMATES \\ FOR POSITIVE SOLUTIONS TO HEAT EQUATION UNDER FINSLER-RICCI FLOW
}

SAJJAD LAKZIAN 


\title{
DIFFERENTIAL HARNACK ESTIMATES FOR POSITIVE SOLUTIONS TO HEAT EQUATION UNDER FINSLER-RICCI FLOW
}

\author{
SAJJAD LAKZIAN
}

\begin{abstract}
We prove first order differential Harnack estimates for positive solutions of the heat equation (in the sense of distributions) under closed Finsler-Ricci flows. We assume suitable Ricci curvature bounds throughout the flow and also assume that the $S$-curvature vanishes along the flow. One of the key tools we use is the Bochner identity for Finsler structures proved by Ohta and Sturm (Adv. Math. 252 (2014), 429-448).
\end{abstract}

\section{Introduction}

In the past few decades, geometric flows and, more notably among them, the Ricci flow have proved very useful in attacking long standing geometry and topology questions. One important application is finding the so-called round (of constant curvature, Einstein, soliton, etc.) metrics on manifolds by homogenizing a given initial metric.

There is also a hope that similar methods can be applied in the Finsler setting. One might hope to find an answer for, for instance, Professor Chern's question about the existence of Finsler-Einstein metrics on every smooth manifold by using a suitable geometric flow resembling the Ricci flow.

In the Finsler setting, there are notions of Ricci and sectional curvatures, and Bao [2007] has proposed an evolution of Finsler structures that in essence shares a great resemblance with the Ricci flow of Riemannian metrics. The flow Bao suggests is $\partial F^{2} / \partial t=-2 F^{2} R$ where $R=\left(1 / F^{2}\right)$ Ric. In terms of the symmetric metric tensor associated with $F$ and Akbarzadeh's Ricci tensor, this flow takes the form of $\partial g_{i j} / \partial t=-2 \mathrm{Ric}_{i j}$ which is the familiar Ricci flow.

The notion of Finsler-Ricci flow is very recent and very little has been done about it. Some partial results regarding the existence and uniqueness of such flows

Lakzian is supported by the Hausdorff postdoctoral fellowship at the Hausdorff Center for Mathematics, University of Bonn, Germany.

MSC2010: primary 35K55; secondary 53C21.

Keywords: Finsler-Ricci flow, differential Harnack, gradient estimate, weighted Ricci curvature, heat equation, curvature-dimension. 
are obtained in [Azami and Razavi 2013]. Also, the solitons of this flow have been studied in [Bidabad and Yarahmadi 2014]. Our focus in these notes will be to consider a positive solution of the heat equation (in the sense of distributions) under Finsler-Ricci flow and prove first order differential Harnack estimates that are similar to those in the Riemannian case (see [Liu 2009; Sun 2011]). The key tools we use are the Bochner identity for Finsler metrics (pointwise and in the sense of distributions) proven by Ohta and Sturm [2014] and, as is customary in such estimates, the maximum principle.

We should mention that, in this paper, we are not dealing with the existence and Sobolev regularity of such solutions (which is very important and extremely delicate - for example, in the static case, solutions will be $C^{2}$ if and only if the structure is Riemannian). For existence and regularity in the static case see [Ohta and Sturm 2009]. Our main theorem is the following.

Theorem 1.1. Let $\left(M^{n}, F(t)\right), t \in[0, T]$ be a closed Finsler-Ricci flow. Suppose there is a real number $K \in \mathbb{R}$ and positive real numbers $K_{1}$ and $K_{2}$ such that, for all $t \in[0, T]$,

(i) $-K_{1} \leq\left(\operatorname{Ric}_{i j}(\boldsymbol{v})\right)_{i, j=1}^{n} \leq K_{2}$ as quadratic forms on $T_{x} M$ for all $\boldsymbol{v} \in T_{x} M \backslash\{0\}$, in any coordinate system, $\left\{\partial / \partial x_{i}\right\}$, that is orthonormal with respect to $g_{v}$, and

(ii) $\boldsymbol{S}$-curvature vanishes (see Section 2.2.7).

Let $u(x, t) \in L^{2}\left([0, T], H^{1}(M)\right) \cap H^{1}\left([0, T], H^{-1}(M)\right)$ be a positive global solution (in the sense of distributions) of the heat equation under Finsler-Ricci flow; i.e., for any test function $\phi \in C^{\infty}(M)$ and for all $t \in[0, T]$,

$$
\int_{M} \phi \partial_{t} u(t, \cdot) d m=-\int_{M} D \phi(\nabla u(t, \cdot)) d m d t .
$$

Then, $u$ satisfies

$$
F^{2}(\nabla(\log u)(t, x))-\theta \partial_{t}(\log u)(t, x) \leq \frac{n \theta^{2}}{t}+\frac{n \theta^{3} C_{1}}{\theta-1}+n^{3 / 2} \theta^{2} \sqrt{C_{2}},
$$

for any $\theta>1$ and where

$$
C_{1}=K_{1} \quad \text { and } \quad C_{2}=\max \left\{K_{1}^{2}, K_{2}^{2}\right\} .
$$

Remark 1.2. Our results can be applied to any Finsler-Ricci flow of Berwald metrics on closed manifolds, since the $\boldsymbol{S}$-curvature vanishes for Berwald metrics (for example, see [Ohta 2011]).

We will note that it might be possible to obtain stronger results with fewer curvature bound conditions by using different methods such as Nash-Moser iteration (as is done by Xia [2014] for harmonic functions in the static case). 
Integrating the differential Harnack inequalities, in a standard manner, leads to Harnack-type inequalities.

Corollary 1.3. Let $(M, F(t)), t \in[0, T]$ be as in Theorem 1.1. Then for any two points $\left(x, t_{1}\right),\left(y, t_{2}\right) \in M \times(0, T]$ with $t_{1}<t_{2}$, we get

$u\left(x, t_{1}\right) \leq u\left(y, t_{2}\right)\left(\frac{t_{2}}{t_{1}}\right)^{2 n \epsilon} \exp \left\{\int_{0}^{1} \frac{\left.\epsilon F^{2}\left(\gamma^{\prime}(s)\right)\right|_{\tau}}{2\left(t_{2}-t_{1}\right)} d s+C(n, \epsilon)\left(t_{2}-t_{1}\right)\left(C_{1}+\sqrt{C_{2}}\right)\right\}$,

whenever $\epsilon>1 / 2$, and for $C$ depending on $n$ and $\epsilon$ only, and where the dependencies of $C_{1}$ and $C_{2}$ on our parameters are as in Theorem 1.1. Here $\gamma$ is a curve joining $x$ and $y$, with $\gamma(1)=x$ and $\gamma(0)=y$, and $\left.F\left(\gamma^{\prime}(s)\right)\right|_{\tau}$ is the speed of $\gamma$ at time $\tau=(1-s) t_{2}+s t_{1}$.

The organization of this paper is as follows: in Section 2, we first briefly review some facts and results about differential Harnack estimates in the Riemannian setting and about Finsler geometry; in Section 3, we present lemmas and computations that we need in order to obtain a useful parabolic partial differential inequality; and in Section 4, we will complete the proof of our main theorem.

\section{Background}

\subsection{Differential Harnack estimates for heat equations in Riemannian Ricciflow.} The Ricci flow equation, $\partial g / \partial t=-2$ Ric, was first proposed by Richard Hamilton in his seminal paper [1982]. Ricci flow is a heat-type quasilinear partial differential equation but, as is well-known, it enjoys a short-time existence and uniqueness theorem (see [Hamilton 1982]) and has been the key tool in proving the Poincaré and geometrization conjectures.

The gradient estimates for solutions of parabolic equations under Ricci flow are a very important part of Ricci flow theory. Perelman in his groundbreaking work [2002] proves such estimates for the conjugate heat equation; he then benefited from these estimates in the analysis of his $\mathcal{W}$-entropy functional. Since then there have been many important results in this direction (for both heat equation and conjugate heat equation) in, for example, [Kuang and Zhang 2008; Bailesteanu et al. 2010; Cao et al. 2013; Cao and Hamilton 2009; Cao 2008], to name a few.

Since our proof, in spirit, is closer to ones in Liu [2009] and Sun [2011], we will only mention their result without commenting on the other literature in this direction. Their estimates for positive solutions of the heat equation under a closed Ricci flow can be stated as follows.

Theorem [Liu 2009; Sun 2011]. Let $(M, g(t)) ; t \in[0, T]$ be a closed Ricci flow solution with $-K_{1} \leq$ Ric $\leq K_{2}\left(K_{1}, K_{2}>0\right)$ along the flow. For $u(x, t)$, a positive solution of the heat equation $\left(\Delta_{g(t)}-\partial_{t}\right) u(x, t)=0$, one has the first order 
gradient estimate

$$
\frac{|\nabla u(x, t)|^{2}}{u^{2}(x, t)}-\theta \frac{\partial_{t} u(x, t)}{u(x, t)} \leq \frac{n \theta^{2}}{t}+\frac{n \theta^{3} K_{1}}{\theta-1}+n^{\frac{3}{2}} \theta^{2}\left(K_{1}+K_{2}\right),
$$

where $\theta>1$.

Their method of proof is to take $f=\log u$ and

$$
\alpha:=t\left(\frac{|\nabla u(x, t)|^{2}}{u^{2}(x, t)}-\theta \frac{\partial_{t} u(x, t)}{u(x, t)}\right)=t\left(|\nabla f|^{2}-\theta \partial_{t} f\right)
$$

and apply the maximum principle to the parabolic partial differential inequality

$$
\left(\Delta_{g(t)}-\partial_{t}\right) \alpha+2 D f(\nabla \alpha) \geq-\frac{\alpha}{t}+\frac{t}{n}\left(|\nabla f|^{2}-\partial_{t} f\right)^{2}-2 \theta K_{1} t|\nabla f|^{2}-t \theta^{2} n^{2}\left(K_{1}+K_{2}\right)^{2} .
$$

This is the method that we will adopt throughout the paper.

\subsection{Finsler structures.}

2.2.1. Finsler metric. Let $M$ be a $C^{\infty}$-connected manifold. A Finsler structure on $M$ consists of a $C^{\infty}$ Finsler norm $F: T M \rightarrow \mathbb{R}$ satisfying the following conditions:

(F1) $F$ is $C^{\infty}$ on $T M \backslash 0$.

(F2) $F$ restricted to the fibers is positively 1 -homogeneous.

(F3) For any nonzero tangent vector $\boldsymbol{y} \in T M$, the approximated symmetric metric tensor defined by

$$
g_{\boldsymbol{y}}(\boldsymbol{u}, \boldsymbol{v}):=\left.\frac{1}{2} \frac{\partial^{2}}{\partial s \partial t} F^{2}(\boldsymbol{y}+s \boldsymbol{u}+t \boldsymbol{v})\right|_{s=t=0}
$$

is positive definite.

2.2.2. Cartan tensor. One way to measure the nonlinearity of a Finsler structure is to introduce the so-called Cartan tensor defined by

$$
C_{\boldsymbol{y}}: \otimes^{3} T M \rightarrow \mathbb{R}, \quad C_{\boldsymbol{y}}(\boldsymbol{u}, \boldsymbol{v}, \boldsymbol{w}):=\frac{1}{2} \frac{d}{d t}\left[g_{\boldsymbol{y}+t \boldsymbol{w}}(\boldsymbol{u}, \boldsymbol{v})\right] .
$$

2.2.3. Legendre transform. In order to define the gradient of a function, we need the Legendre transform, $\mathcal{L}^{*}: T^{*} M \rightarrow T M$. For $\omega \in T^{*} M$, let $\mathcal{L}^{*}(\omega)$ be the unique vector $\boldsymbol{y} \in T M$ such that

$$
\omega(\boldsymbol{y})=F^{*}(\omega)^{2} \text { and } F(\boldsymbol{y})=F^{*}(\omega),
$$

where $F^{*}$ is the dual norm to $F$.

For a smooth function $u: M \rightarrow \mathbb{R}$, the gradient of $u$ is $\nabla u(x):=\mathcal{L}^{*}(D u(x))$. 
2.2.4. Geodesic spray, Chern connection and curvature tensor. It is easy to see that the geodesic spray in the Finsler setting is of the form $\boldsymbol{G}=y^{i} \partial / \partial x_{i}$ $2 G^{i}(x, \boldsymbol{y}) \partial / \partial y^{i}$, where

$$
G^{i}(x, \boldsymbol{y})=\frac{1}{4} g_{\boldsymbol{y}}^{i k}\left\{2 \frac{\partial\left(g_{\boldsymbol{y}}\right)_{j k}}{\partial x_{l}}-\frac{\partial\left(g_{\boldsymbol{y}}\right)_{j l}}{\partial x_{k}}\right\} y^{j} y^{l} .
$$

The nonlinear connection that we will be using in this work is the Chern connection, the connection coefficients of which are given by

$$
\Gamma_{j k}^{i}=\Gamma_{k j}^{i}:=\frac{1}{2} g^{i l}\left\{\frac{\partial g_{l j}}{\partial x_{k}}-\frac{\partial g_{j k}}{\partial x_{l}}+\frac{\partial g_{k l}}{\partial x_{j}}-\frac{\partial g_{l j}}{\partial y^{r}} G_{k}^{r}+\frac{\partial g_{j k}}{\partial y^{r}} G_{l}^{r}-\frac{\partial g_{k l}}{\partial y^{r}} G_{j}^{r}\right\},
$$

where $G_{j}^{i}:=\partial G^{i} / \partial y^{j}$ and $g$ is in fact $g_{\boldsymbol{y}}$.

For Berwald metrics, the geodesic coefficients $G^{i}$ are quadratic in terms of $y$ (by definition) which immensely simplifies the formula for connection coefficients. In fact for Berwald metrics we have $\Gamma_{j k}^{i}=\partial^{2} G^{i} / \partial y^{j} \partial y^{k}$.

Similar to the Riemannian setting, one uses the Chern connection (and the associated covariant differentiation) to define the curvature tensor

$$
R^{V}(X, Y) Z:=\left[\nabla_{X}^{V}, \nabla_{Y}^{V}\right] Z-\nabla_{[X, Y]}^{V} Z,
$$

which, of course, depends on a nonzero vector field $V$.

2.2.5. Flag and Ricci curvatures. Flag curvature is defined similar to the sectional curvature in the Riemannian setting. For a fixed flag pole $v \in T_{x} M$ and for $\boldsymbol{w} \in T_{x} M$, the flag curvature is defined by

$$
\mathcal{K}^{v}(\boldsymbol{v}, \boldsymbol{w}):=\frac{g_{\boldsymbol{v}}\left(R^{v}(\boldsymbol{v}, \boldsymbol{w}) \boldsymbol{w}, \boldsymbol{v}\right)}{g_{\boldsymbol{v}}(\boldsymbol{v}, \boldsymbol{v}) g_{\boldsymbol{v}}(\boldsymbol{w}, \boldsymbol{w})-g_{\boldsymbol{v}}(\boldsymbol{v}, \boldsymbol{w})^{2}} .
$$

The Ricci curvature is then the trace of the flag curvature, i.e.,

$$
\operatorname{Ric}(\boldsymbol{v}):=F^{2}(\boldsymbol{v}) \sum_{i=1}^{n-1} \mathcal{K}^{\boldsymbol{v}}\left(\boldsymbol{v}, \boldsymbol{e}_{i}\right),
$$

where $\left\{\boldsymbol{e}_{1}, \ldots, \boldsymbol{e}_{n-1}, \frac{\boldsymbol{v}}{\boldsymbol{F}(\boldsymbol{v})}\right\}$ constitutes a $g_{\boldsymbol{v}}$-orthonormal basis of $T_{x} M$.

2.2.6. Akbarzadeh's Ricci tensor. Akbarzadeh's Ricci tensor is defined by

$$
\operatorname{Ric}_{i j}:=\frac{\partial^{2}}{\partial y^{i} \partial y^{j}}\left(\frac{\mathrm{Ric}}{2}\right) .
$$

It can be shown that the scalar Ricci curvature, Ric, and Akbarzadeh's Ricci tensor, $\mathrm{Ric}_{i j}$, have the same geometrical implications. For further details regarding this tensor, see [Bao and Robles 2004]. 
2.2.7. $S$-curvature. Associated with any Finsler structure, there is one canonical measure, called the Busemann-Hausdorff measure, which is given by

$$
d V_{F}:=\sigma_{F}(x) d x_{1} \wedge \cdots \wedge d x_{n},
$$

where $\sigma_{F}(x)$ is the volume ratio

$$
\sigma_{F}(x):=\frac{\operatorname{vol}\left(B_{\mathbb{R}^{n}}(1)\right)}{\operatorname{vol}\left(\boldsymbol{y} \in T_{x} M: F(\boldsymbol{y})<1\right)} .
$$

The set whose volume appears in the denominator of (17) is called the indicatrix, and there is often no known way to express its volume in terms of $F$.

The $S$-curvature, which is another measure of nonlinearity, is then defined by

$$
\boldsymbol{S}(\boldsymbol{y}):=\frac{\partial G^{i}}{\partial y^{i}}(x, \boldsymbol{y})-y^{i} \frac{\partial}{\partial x_{i}}\left(\ln \sigma_{F}(x)\right) .
$$

For more details, see [Shen 2004], for example.

2.2.8. Hessian, divergence and Laplacian. The Hessian in a Finsler structure is defined by

$$
\operatorname{Hess}(u)(X, Y):=X Y(u)-\nabla_{X}^{\nabla u} Y(u)=g_{\nabla u}\left(\nabla_{X}^{\nabla u} \nabla u, Y\right) .
$$

As usual, for a twice differentiable function $u$,

$$
\operatorname{Hess}(u)\left(\frac{\partial}{\partial x_{i}}, \frac{\partial}{\partial x_{j}}\right)=\frac{\partial^{2} u}{\partial x_{i} \partial x_{j}}-\Gamma_{i j}^{k} \frac{\partial u}{\partial x_{k}}
$$

For a smooth measure $\mu=e^{-\Psi} d x_{1} \wedge \cdots \wedge d x_{n}$ and a vector field $V$, the divergence is defined by

$$
\operatorname{div}_{\mu} V:=\sum_{i=1}^{n}\left(\frac{\partial V_{i}}{\partial x_{i}}-V_{i} \frac{\partial \Psi}{\partial x_{i}}\right)
$$

Now, using this divergence, one can define the distributional Laplacian of a function $u \in H^{1}(M)$ by $\Delta u:=\operatorname{div}_{\mu}(\nabla u)$, i.e.,

$$
\int_{M} \phi \Delta u d \mu:=-\int_{M} D \phi(\nabla u) d \mu
$$

for $\phi \in C^{\infty}(M)$.

The Finsler distributional Laplacian is nonlinear but fortunately there is a way to relate it to the trace of the Hessian by adding an $\boldsymbol{S}$-curvature term. Indeed, one has

$$
\Delta u=\operatorname{tr}_{\nabla u} \operatorname{Hess}(u)-\boldsymbol{S}(\nabla u) .
$$

For a proof of (23), see for instance [Wu and Xin 2007]. 
2.3. Weighted Ricci curvature and Bochner-Weitzenböck formula. The notion of the weighted Ricci curvature, $\mathrm{Ric}_{N}$, of a Finsler structure equipped with a measure $\mu$ was introduced by Ohta [2009]. Take a unit vector $v \in T_{x} M$ and let $\gamma:[-\epsilon,+\epsilon] \rightarrow M$ be a short geodesic whose velocity at time $t=0$ is $\dot{\gamma}(0)=\boldsymbol{v}$. Decompose the measure $\mu$ along $\gamma$ with respect to the Riemannian volume form; i.e., let $\mu=e^{-\Psi} d \operatorname{vol}_{\dot{\gamma}}$. Then

$$
\operatorname{Ric}_{n}(v):= \begin{cases}\operatorname{Ric}(v)+(\Psi \circ \gamma)^{\prime \prime}(0) & \text { if }(\Psi \circ \gamma)^{\prime}(0)=0 \\ -\infty & \text { otherwise }\end{cases}
$$

Also $\operatorname{Ric}_{N}(\lambda \boldsymbol{v}):=\lambda^{2} \operatorname{Ric}_{N}(\boldsymbol{v})$ for $\lambda \geq 0$.

It is proven in [Ohta 2009] that the curvature bound $\mathrm{Ric}_{N} \geq K F^{2}$ is equivalent to the Lott-Villani-Sturm $C D(K, N)$ condition.

Using the weighted Ricci curvature bounds, Ohta and Sturm [2014] proved the Bochner-Weitzenböck formulae (both pointwise and integrated versions) for Finsler structures. For $u \in C^{\infty}(M)$, the pointwise version of the identity and inequality are

$$
\begin{gathered}
\Delta^{\nabla u}\left(\frac{F^{2}(\nabla u)}{2}\right)-D(\Delta u)(\nabla u)=\operatorname{Ric}_{\infty}(\nabla u)+\left\|\nabla^{2} u\right\|_{H S(\nabla u)}^{2} \quad \text { (identity), } \\
\Delta^{\nabla u}\left(\frac{F^{2}(\nabla u)}{2}\right)-D(\Delta u)(\nabla u) \geq \operatorname{Ric}_{N}(\nabla u)+\frac{(\Delta u)^{2}}{N} \quad \text { (inequality). }
\end{gathered}
$$

\section{Estimates}

In this section we will gather all the required lemmas and estimates that will be needed to apply the maximum principle.

Evolution of the Legendre transform. Since in the Finsler setting the gradient is nonlinear and depends on the Legendre transform, we will need to know the evolution of the Legendre transform under Finsler-Ricci flow.

Let $(M, F)$ be a Finsler structure evolving under Finsler-Ricci flow. Then the inverse of the Legendre transform is defined by

$$
\left(\mathcal{L}^{*}\right)^{-1}: T M \rightarrow T^{*} M, \quad\left(\mathcal{L}^{*}\right)^{-1}(x, y)=(x, p), \quad \text { where } p_{i}=g_{i j}(x, y) y^{j} .
$$

To explicitly formulate the Legendre transform, we have, for any given $\omega \in T_{x}^{*} M$, that $\mathcal{L}^{*}(\omega)=y \in T_{x} M$, where $y$ is the unique solution to the nonlinear system

$$
g(x, y)_{k 1} \cdot y^{1}+\cdots+g(x, y)_{k n} \cdot y^{n}=\omega_{k}, \quad \text { for } k=1, \ldots, n,
$$


or, in the matrix form,

$$
g(\boldsymbol{y}) \boldsymbol{y}=\omega .
$$

Lemma 3.1. Let $(M, F(t))$ be a Finsler structure evolving by Finsler-Ricci flow. Then the Legendre transform $\mathcal{L}^{*}: T^{*} M \rightarrow T M$ satisfies

$$
\partial_{t} \mathcal{L}^{*}=2 \operatorname{Ric}_{j}^{i} \mathcal{L}^{*}
$$

i.e., for any fixed 1 -form $\omega$ with $\mathcal{L}^{*}(\omega)=\boldsymbol{y}=y^{i} \partial / \partial x_{i} \in T M$, we have

$$
\partial_{t} y^{i}=2 \operatorname{Ric}_{r}^{i} y^{r},
$$

where $\operatorname{Ric}_{r}^{i}:=g^{i j} \operatorname{Ric}_{j r}$.

Proof. Fix $\omega$ and differentiate both sides of (31) with respect to $t$ to get

$$
\left[\partial_{t} g(\boldsymbol{y})\right] \boldsymbol{y}+g(\boldsymbol{y}) \partial_{t} \boldsymbol{y}=0 .
$$

Therefore,

$$
\partial_{t} \boldsymbol{y}=-g(\boldsymbol{y})^{-1} \partial_{t} g(\boldsymbol{y}) \boldsymbol{y} .
$$

Expanding the right-hand side of (35), we have, for every $i$,

$$
\begin{aligned}
\partial_{t} y^{i} & =-g(\boldsymbol{y})^{i j}\left(\partial_{t} g(\boldsymbol{y})\right)_{j r} y^{r} \\
& =2 g(\boldsymbol{y})^{i j} \operatorname{Ric}_{j r}(\boldsymbol{y}) y^{r}-g(\boldsymbol{y})^{i j}\left(\frac{\partial g_{j r}}{\partial y^{k}} \partial_{t} y^{k}\right) y^{r} \\
& =2 \operatorname{Ric}_{r}^{i}(\boldsymbol{y}) y^{r} .
\end{aligned}
$$

Notice that the second term in the second line of (36) vanishes by Euler's theorem.

Evolution of $\boldsymbol{F}^{\mathbf{2}}(\nabla \boldsymbol{f})$. One crucial step in the proof of the gradient estimates is to be able to estimate the evolution of the term $F^{2}(\nabla f)$.

Lemma 3.2. Let $(M, F(t))$ be a time-dependent Finsler structure. Then

$$
\partial_{t}\left[F^{2}(\nabla f)\right]=2 g^{i j}(D f)\left[\partial_{t} f\right]_{i} f_{j}+\left[\partial_{t} g^{i j}\right](D f) f_{i} f_{j} .
$$

Proof. Simple differentiation gives

$$
\begin{aligned}
\partial_{t}\left[F^{2}(\nabla f)\right] & =\partial_{t}\left[F^{*}(D f)^{2}\right] \\
& =\partial_{t}\left[g^{i j}(D f) f_{i} f_{j}\right] \\
& =2 g^{i j}(D f)\left[\partial_{t} f\right]_{i} f_{j}+\partial_{t}\left[g^{i j}(D f)\right] f_{i} f_{j} .
\end{aligned}
$$

Expanding the second term of the last line in (38), we have

$$
\partial_{t}\left[g^{i j}(D f)\right] f_{i} f_{j}=\left[\partial_{t} g^{i j}\right](D f) f_{i} f_{j}+\frac{\partial g^{i j}}{\partial y^{k}} \partial_{t} y^{k}(D f) f_{i} f_{j} .
$$


Using Euler's theorem, the second term of the right-hand side of (39) vanishes.

Lemma 3.3. Suppose $F$ is evolving by the Finsler-Ricci flow equation. Then

$$
\partial_{t}\left[F^{2}(\nabla f)\right]=2 D\left(\partial_{t} f\right)(\nabla f)+2 \operatorname{Ric}^{i j}(D f) f_{i} f_{j} .
$$

Proof. It is standard to see that under Finsler-Ricci flow, we have

$$
\partial_{t} g^{i j}=2 \mathrm{Ric}^{i j},
$$

where, as before, $\operatorname{Ric}^{i j}:=g^{i r} g^{j s} \operatorname{Ric}_{r s}$.

\section{Proof of main theorem}

In this section we will complete the proof of our main theorem. Throughout the rest of these notes, we consider a solution $u$ of the heat equation. The Laplacian, gradient and Legendre transform are all with respect to $V:=\nabla u$ and are valid on $M_{u}:=\{x \in M: \nabla u(x) \neq 0\}$.

Let $\sigma(t, x)=t \partial_{t} f(t, x)$ where $f=\log u$. Then we have $g_{\nabla f}=g_{V}$. Let

$$
\alpha(t, x):=t\left\{F^{2}(\nabla f(t, x))-\theta \partial_{t} f(t, x)\right\}=t F^{2}(\nabla f(t, x))-\theta \sigma .
$$

Lemma 4.1. In the sense of distributions, $\sigma(t, x)$ satisfies the parabolic differential equality

$$
\Delta \sigma-\partial_{t} \sigma+\frac{\sigma}{t}+2 D \sigma(\nabla f)=t\left\{-2 \operatorname{Ric}^{i j}(\nabla f) f_{i} f_{j}-2(\operatorname{Ric})^{k l}(\nabla f) f_{k l}\right\} .
$$

Proof. We first note that, for any nonnegative test function $\phi \in H^{1}([0, T] \times M)$ whose support is included in the domain of the local coordinate,

$$
\partial_{t}(D(t \phi)(\nabla f))=D\left(\partial_{t}(t \phi)\right)(\nabla f)+D(t \phi)\left(\nabla\left(\partial_{t} f\right)\right)+2(\operatorname{Ric})^{i j}(\nabla f) \frac{\partial(t \phi)}{\partial x_{i}} \frac{\partial f}{\partial x_{j}} .
$$

Indeed,

$$
\begin{aligned}
\partial_{t}(D(t \phi) & (\nabla f)) \\
& =D\left(\partial_{t}(t \phi)\right)(\nabla f)+D(t \phi)\left(\partial_{t}\left(\mathcal{L}^{*}(D f)\right)\right. \\
& =D\left(\partial_{t}(t \phi)\right)(\nabla f)+D(t \phi)\left(\partial_{t}\left(\mathcal{L}^{*}\right)(D f)+\mathcal{L}^{*}\left(D \partial_{t} f\right)\right) \\
& =D\left(\partial_{t}(t \phi)\right)(\nabla f)+D(t \phi)\left(\partial_{t}\left(\mathcal{L}^{*}\right)(D f)\right)+D(t \phi)\left(\mathcal{L}^{*}\left(D \partial_{t} f\right)\right) \\
& =D\left(\partial_{t}(t \phi)\right)(\nabla f)+D(t \phi)\left(\nabla\left(\partial_{t} f\right)\right)+2 g^{s j}(\operatorname{Ric})_{s}^{i}(\nabla f) \frac{\partial(t \phi)}{\partial x_{i}} \frac{\partial f}{\partial x_{j}} \\
& =D\left(\partial_{t}(t \phi)\right)(\nabla f)+D(t \phi)\left(\nabla\left(\partial_{t} f\right)\right)+2(\operatorname{Ric})^{i j}(\nabla f) \frac{\partial(t \phi)}{\partial x_{i}} \frac{\partial f}{\partial x_{j}} .
\end{aligned}
$$


That is,

$-D(t \phi)\left(\nabla\left(\partial_{t} f\right)\right)=-\partial_{t}(D(t \phi)(\nabla f))+D\left(\partial_{t}(t \phi)\right)(\nabla f)+2(\operatorname{Ric})^{i j}(\nabla f) \frac{\partial(t \phi)}{\partial x_{i}} \frac{\partial f}{\partial x_{j}}$.

Multiplying the left-hand side of (43) by $\phi$, integrating and then substituting (46), we get

$$
\begin{aligned}
\boldsymbol{A} & =\int_{0}^{T} \int_{M}\left\{-D \phi(\nabla \sigma)+\partial_{t} \phi \cdot \sigma+\frac{\phi \sigma}{t}+2 \phi D \sigma(\nabla f)\right\} d m d t \\
= & \int_{0}^{T} \int_{M}\left\{-D(t \phi)\left(\nabla\left(\partial_{t} f\right)\right)+\partial_{t}(t \phi) \partial_{t} f+2 t \phi D\left(\partial_{t} f\right)(\nabla f)\right\} d m d t \\
= & \int_{0}^{T} \int_{M}\left\{D\left(\partial_{t}(t \phi)\right)(\nabla f)+\partial_{t}(t \phi)\left(\Delta f+F^{2}(\nabla f)\right)\right. \\
& \left.+2(\text { Ric })^{i j}(\nabla f) \frac{\partial(t \phi)}{\partial x_{i}} \frac{\partial f}{\partial x_{j}}+2 t \phi D\left(\partial_{t} f\right)(\nabla f)\right\} d m d t .
\end{aligned}
$$

Using the estimates we have obtained for $\partial_{t}\left[F(\nabla f)^{2}\right]$ in Lemmas 3.2 and 3.3, we arrive at

$$
\begin{aligned}
\boldsymbol{A}= & \int_{0}^{T} \int_{M}\left\{D\left(\partial_{t}(t \phi)\right)(\nabla f)+\partial_{t}(t \phi)(\Delta f)+\partial_{t}(t \phi)\left(F^{2}(\nabla f)\right)\right. \\
& \left.+2(\operatorname{Ric})^{i j}(\nabla f) \frac{\partial(t \phi)}{\partial x_{i}} \frac{\partial f}{\partial x_{j}}+2 t \phi D\left(\partial_{t} f\right)(\nabla f)\right\} d m d t \\
= & \int_{0}^{T} \int_{M}\left\{\partial_{t}(t \phi)\left(F^{2}(\nabla f)\right)+2(\operatorname{Ric})^{i j}(\nabla f) \frac{\partial(t \phi)}{\partial x_{i}} \frac{\partial f}{\partial x_{j}}+t \phi \partial_{t}\left[F(\nabla f)^{2}\right]\right. \\
\left.-2 t \phi \operatorname{Ric}^{i j}(\nabla f) f_{i} f_{j}\right\} d m d t & \\
= & \int_{0}^{T} \int_{M} t \phi\left\{-2 \operatorname{Ric}^{i j}(\nabla f) f_{i} f_{j}-2 \operatorname{Ric}^{i j}(\nabla f) f_{i j}\right\} d m d t .
\end{aligned}
$$

Notice that Euler's theorem has been used in the last line of (48).

Now we can compute a parabolic partial differential inequality for $\alpha(t, x)$ with a similar left-hand side.

Lemma 4.2. In the sense of distributions, $\alpha(t, x)$ satisfies

$$
\Delta^{V} \alpha+2 D \alpha(\nabla f)-\partial_{t} \alpha+\frac{\alpha}{t}=\boldsymbol{B}
$$

where

$$
\boldsymbol{B}=\theta\left(2 t \operatorname{Ric}^{i j}(\nabla f) f_{i} f_{j}+2 t \operatorname{Ric}^{k l}(\nabla f) f_{k l}\right)
$$

$$
+2 t \operatorname{Ric}(\nabla f)+2 t\left\|\nabla^{2} f\right\|_{H S(\nabla f)}^{2}-2 t \operatorname{Ric}^{i j}(\nabla f) f_{i} f_{j} .
$$


Proof. For a nonnegative test function $\phi$, one computes

$$
\begin{gathered}
\int_{0}^{T} \int_{M}\left\{-D \phi(\nabla \alpha)+\partial_{t} \phi \alpha+\frac{\phi \alpha}{t}+2 \phi D \alpha(\nabla f)\right\} d m d t \\
=-\theta \boldsymbol{A}+\int_{0}^{T} \int_{M}\left\{-t D \phi\left(\nabla\left(F^{2}(\nabla f)\right)+\partial_{t} \phi\left(t F^{2}(\nabla f)\right)\right.\right. \\
\left.+\phi\left(F^{2}(\nabla f)\right)+2 t \phi D\left(F^{2}(\nabla f)\right)(\nabla f)\right\} d m d t
\end{gathered}
$$

where $\boldsymbol{A}$ is as in (48).

Again using the estimates for $\partial_{t}\left[F(\nabla f)^{2}\right]$ (as in Lemmas 3.2 and 3.3), we arrive at

$$
\begin{gathered}
\int_{0}^{T} \int_{M}\left\{-D \phi(\nabla \alpha)+\partial_{t} \phi \cdot \alpha+\frac{\phi \alpha}{t}+2 \phi D \alpha(\nabla f)\right\} d m d t \\
=-\theta \boldsymbol{A}+\int_{0}^{T} \int_{M}\left\{-t D \phi\left(\nabla\left(F^{2}(\nabla f)\right)\right)-\phi \cdot t \partial_{t}\left(F^{2}(\nabla f)\right)\right. \\
\left.+2 t \phi D\left(F^{2}(\nabla f)\right)(\nabla f)\right\} d m d t \\
=-\theta \boldsymbol{A}+\int_{0}^{T} \int_{M}\left\{-t D \phi\left(\nabla\left(F^{2}(\nabla f)\right)\right)-2 t \phi D\left(\partial_{t} f\right)(\nabla f)\right. \\
=-\theta \boldsymbol{A}+\int_{0}^{T} \int_{M}\left\{-t D \phi\left(\nabla\left(F^{2}(\nabla f)\right)\right)-2 t \phi D(\Delta f)(\nabla f)\right. \\
-2 t \phi D\left(F^{2}(\nabla f)\right)(\nabla f)-2 t \phi \operatorname{Ric}^{i j} f_{i} f_{j} \\
\left.+2 t \phi D\left(F^{2}(\nabla f)\right)(\nabla f)\right\} d m d t \\
=-\theta \boldsymbol{A}+\int_{0}^{T} \int_{M}\left\{-t D \phi\left(\nabla\left(F^{2}(\nabla f)\right)\right)\right. \\
\left.-2 t \phi D(\Delta f)(\nabla f)-2 t \phi \operatorname{Ric}^{i j}(\nabla f) f_{i} f_{j}\right\} d m d t .
\end{gathered}
$$


By applying the Bochner-Weitzenböck formula (proven in [Ohta and Sturm

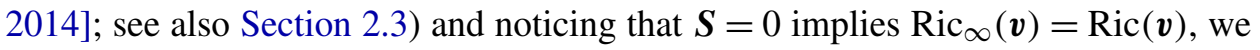
can continue as follows:

$$
\begin{aligned}
-\theta \boldsymbol{A} & +\int_{0}^{T} \int_{M}\left\{-t D \phi\left(\nabla\left(F^{2}(\nabla f)\right)\right)-2 t \phi D(\Delta f)(\nabla f)-2 t \phi \operatorname{Ric}^{i j} f_{i} f_{j}\right\} d m d t \\
& =-\theta \boldsymbol{A}+\int_{0}^{T} \int_{M} \phi\left\{2 t \operatorname{Ric}(\nabla f)+2 t\left\|\nabla^{2} f\right\|_{H S(\nabla f)}^{2}-2 t \operatorname{Ric}^{i j}(\nabla f) f_{i} f_{j}\right\} d m d t .
\end{aligned}
$$

Now, substituting $\boldsymbol{A}$ from (47), we have

$$
\begin{aligned}
\boldsymbol{B}=\theta\left(2 t \operatorname{Ric}^{i j}(\nabla f) f_{i} f_{j}+2 t \operatorname{Ric}^{k l}(\nabla f)\right. & \left.f_{k l}\right)+2 t \operatorname{Ric}(\nabla f) \\
& +2 t\left\|\nabla^{2} f\right\|_{H S(\nabla f)}^{2}-2 t \operatorname{Ric}^{i j}(\nabla f) f_{i} f_{j} .
\end{aligned}
$$

Proof of Theorem 1.1. Assume the curvature bounds given in the statement of Theorem 1.1, and assume that the $\boldsymbol{S}$-curvature vanishes. The constants obtained below all depend on our curvature bounds and the ellipticity of the flow.

Let's start with $B(t, x)$ :

$$
\begin{aligned}
B(t, x)=\theta\left(2 t \operatorname{Ric}^{i j}(\nabla f) f_{i} f_{j}+2 t \operatorname{Ric}^{k l}(\nabla f)\right. & \left.f_{k l}\right)+2 t \operatorname{Ric}(\nabla f) \\
& +2 t\left\|\nabla^{2} f\right\|_{H S(\nabla f)}^{2}-2 t \operatorname{Ric}^{i j}(\nabla f) f_{i} f_{j} .
\end{aligned}
$$

Young's inequality tells us that

$$
\left|\operatorname{Ric}^{k l} f_{k l}\right| \leq \frac{\theta}{2}\left(\operatorname{Ric}^{k l}\right)^{2}+\frac{1}{2 \theta} f_{k l}^{2},
$$

and therefore

$$
2 \theta t\left|\operatorname{Ric}^{k l} f_{k l}\right| \leq t \theta^{2}\left(\operatorname{Ric}^{k l}\right)^{2}+t f_{k l}^{2} .
$$

Pick a normal coordinate system with respect to $g_{\nabla f}$, with $\nabla f(x)=\partial / \partial x_{1}$ as well as $\Gamma_{i j}^{1}(\nabla f(x))=0$ for all $i, j$. Then

$$
\operatorname{Ric}^{i j}(\nabla f)=\operatorname{Ric}_{i j}(\nabla f), \quad\left\|\nabla^{2} f\right\|_{H S(\nabla f)}^{2}=\sum f_{i j}^{2}, \quad \sum_{i=1}^{n} f_{i i}=\Delta f(x),
$$

and consequently

$$
\begin{array}{r}
B(t, x) \geq 2 t \theta \operatorname{Ric}_{i j}(\nabla f) f_{i} f_{j}-t \sum \theta^{2}\left(\operatorname{Ric}_{k l}\right)^{2}-t \sum f_{k l}^{2} \\
+2 t \operatorname{Ric}(\nabla f)+2 t\left\|\nabla^{2} f\right\|_{H S(\nabla f)}^{2}-2 t \operatorname{Ric}_{i j}(\nabla f) f_{i} f_{j} \\
\geq-2 t \theta K_{1} F^{2}(\nabla f)-2 t K_{1} F^{2}(\nabla f)+t \sum f_{i j}^{2} \\
-t \theta^{2} n^{2} C_{2}+2 t K_{1} F^{2}(\nabla f) .
\end{array}
$$


On the other hand, one computes

$$
\sum f_{i j}^{2} \geq \sum f_{i i}^{2} \geq \frac{1}{n}\left(\sum f_{i i}\right)^{2}=\frac{1}{n}(\Delta f)^{2} .
$$

Hence,

$$
t \sum f_{i j}^{2} \geq \frac{t}{n}(\Delta f)^{2}
$$

Putting all the above estimates together and noting that $\theta>1$, we get

$$
\begin{aligned}
B(t, x) & \geq \frac{t}{n}(\Delta f)^{2}-2 t \theta K_{1} F^{2}(\nabla f)-2 t K_{1} F^{2}(\nabla f)-t \theta^{2} n^{2} C_{2}+2 t K_{1} F^{2}(\nabla f) \\
& \geq \frac{t}{n}(\Delta f)^{2}-2 t \theta K_{1} F^{2}(\nabla f)-t \theta^{2} n^{2} C_{2} .
\end{aligned}
$$

Replacing the term $\Delta f$ with $\left(F(\nabla f)^{2}-\partial_{t} f\right)$, we get the inequality

$$
B(t, x) \geq \frac{t}{n}\left(F(\nabla f)^{2}-\partial_{t} f\right)^{2}-2 t \theta C_{1} F^{2}(\nabla f)-t \theta^{2} n^{2} C_{2},
$$

where

$$
\begin{aligned}
& C_{1}=K_{1}, \\
& C_{2}=\max \left\{K_{1}^{2}, K_{2}^{2}\right\} .
\end{aligned}
$$

This means that

$$
\begin{aligned}
\Delta^{V} \alpha+2 D \alpha(\nabla f) & -\partial_{t} \alpha \\
& \geq-\frac{\alpha}{t}+\frac{t}{n}\left(F(\nabla f)^{2}-\partial_{t} f\right)^{2}-2 t \theta C_{1} F^{2}(\nabla f)-t \theta^{2} n^{2} C_{2} .
\end{aligned}
$$

This inequality is exactly of the form that appears in [Liu 2009], and a computation similar to the one at the end of the proof of [Liu 2009, Theorem 2] (using the quadratic formula and maximum principle) gives the desired result. For the sake of clarity, we will repeat the computation here.

Let

$$
\bar{\alpha}:=\alpha-t \frac{n \theta^{3} C_{1}}{(\theta-1)}-t n^{3 / 2} \theta^{2} \sqrt{C_{2}} .
$$

Suppose the maximum of $\bar{\alpha}$ is attained at $\left(x_{0}, t_{0}\right)$ and suppose $\bar{\alpha}\left(x_{0}, t_{0}\right)>n \theta^{2}$ (which implicitly implies $\left.t_{0}>0\right)$. Therefore, at $\left(x_{0}, t_{0}\right)$, we have

$$
0 \geq\left(\Delta-\partial_{t}\right) \bar{\alpha} \geq\left(\Delta-\partial_{t}\right) \alpha .
$$

Let $w:=F^{2}(\nabla f)$ and $z:=\partial_{t} f$. Then in terms of $w$ and $z$ we have

$$
0 \geq-\frac{\alpha}{t_{0}}+\frac{t_{0}}{n}(w-z)^{2}-2 t_{0} \theta C_{1} w-t_{0} \theta^{2} n^{2} C_{2} .
$$


By the quadratic formula, we get

$$
\begin{aligned}
& \frac{t_{0}}{n}(w-z)^{2}-2 t_{0} \theta C_{1} w \\
& \quad=\frac{t_{0}}{n}\left(\frac{1}{\theta^{2}}(w-\theta z)^{2}+\left(\frac{\theta-1}{\theta}\right)^{2} w^{2}-2 \theta n C_{1} w+2\left(\frac{\theta-1}{\theta^{2}} w\right)(w-\theta z)\right) \\
& \quad \geq \frac{t_{0}}{n}\left(\frac{1}{\theta^{2}}(w-\theta z)^{2}-\frac{\theta^{4} n^{2} C_{1}^{2}}{(\theta-1)^{2}}+2\left(\frac{\theta-1}{\theta^{2}} w\right)(w-\theta z)\right) .
\end{aligned}
$$

Therefore,

$$
\begin{aligned}
0 & \geq \frac{t_{0}}{n \theta^{2}}\left(\frac{\alpha}{t_{0}}\right)^{2}-\frac{\alpha}{t_{0}}-\frac{n \theta^{4} C_{1}^{2}}{(\theta-1)^{2}} t_{0}-t_{0} \theta^{2} n^{2} C_{2}+\frac{2 t_{0}}{n} \frac{\theta-1}{\theta^{2}} F^{2}(\nabla f)\left(\frac{\alpha}{t_{0}}\right) \\
& \geq \frac{t_{0}}{n \theta^{2}}\left(\frac{\alpha}{t_{0}}\right)^{2}-\frac{\alpha}{t_{0}}-\frac{n \theta^{4} C_{1}^{2}}{(\theta-1)^{2}} t_{0}-t_{0} \theta^{2} n^{2} C_{2} .
\end{aligned}
$$

Using the quadratic formula one more time, (66) implies that

$$
\frac{\alpha}{t_{0}} \leq \frac{n \theta^{2}}{t_{0}}+\frac{n \theta^{3} C_{1}}{\theta-1}+n^{\frac{3}{2}} \theta^{2} \sqrt{C_{2}}
$$

which in turn implies

$$
\bar{\alpha}\left(x_{0}, t_{0}\right) \leq n \theta^{2},
$$

and this is a contradiction. Therefore,

$$
F^{2}(\nabla(\log u)(t, x))-\theta \partial_{t}(\log u)(t, x) \leq \frac{n \theta^{2}}{t}+\frac{n \theta^{3} C_{1}}{(\theta-1)}+n^{3 / 2} \theta^{2} \sqrt{C_{2}},
$$

with $C_{1}$ and $C_{2}$ as in (59) and (60).

Proof of Corollary 1.3. From Theorem 1.1, we know that

$$
F^{2}(\nabla(\log u)(t, x))-\theta \partial_{t}(\log u)(t, x) \leq \frac{n \theta^{2}}{t}+C(n, \theta)\left(C_{1}+\sqrt{C_{2}}\right) .
$$

Let $l(s):=\ln u(\gamma(s), \tau(s))=f(\gamma(s), \tau(s))$. Then

$$
\begin{aligned}
\frac{\partial l(s)}{\partial s} & =\left(t_{2}-t_{1}\right)\left(\frac{D f(\dot{\gamma}(s))}{t_{2}-t_{1}}-\partial_{t} f\right) \\
& \leq\left(t_{2}-t_{1}\right)\left(\frac{F(\nabla f) F(\dot{\gamma})}{t_{2}-t_{1}}-\partial_{t} f\right) \\
& \leq\left(t_{2}-t_{1}\right)\left(\frac{\left.\epsilon F^{2}(\dot{\gamma})\right|_{\tau}}{2\left(t_{2}-t_{1}\right)^{2}}+\frac{1}{2 \epsilon} F^{2}(\nabla f)-\partial_{t} f\right) \\
& \leq \frac{\left.\epsilon F^{2}(\dot{\gamma})\right|_{\tau}}{2\left(t_{2}-t_{1}\right)}+\left(t_{2}-t_{1}\right)\left(\frac{2 n \epsilon}{\tau}+C(n, \epsilon)\left(C_{1}+\sqrt{C_{2}}\right)\right) .
\end{aligned}
$$


Integrating this inequality gives

$$
\begin{aligned}
\ln \frac{u\left(x, t_{1}\right)}{u\left(y, t_{2}\right)} & =\int_{0}^{1} \frac{\partial l(s)}{\partial s} d s \\
& \leq \int_{0}^{1} \frac{\left.\epsilon F^{2}(\dot{\gamma})\right|_{\tau}}{2\left(t_{2}-t_{1}\right)} d s+C(n, \epsilon)\left(t_{2}-t_{1}\right)\left(C_{1}+\sqrt{C_{2}}\right)+2 \epsilon n \ln \frac{t_{2}}{t_{1}} .
\end{aligned}
$$

\section{Acknowledgements}

The author would like to thank Professor K.-T. Sturm for the opportunity of working under his supervision as a postdoctoral fellow and for his great insights. The author also would like to thank Professor Christina Sormani for her constant support and encouragement. Many thanks go to the stochastic analysis group in Bonn and also my colleagues Mike Munn and Lashi Bandara.

The author is deeply grateful for valuable comments made and numerous corrections suggested by the anonymous reviewer of this paper.

\section{References}

[Azami and Razavi 2013] S. Azami and A. Razavi, "Existence and uniqueness for solution of Ricci flow on Finsler manifolds", Int. J. Geom. Methods Mod. Phys. 10:3 (2013), Article ID \#1250091. MR 3011654 Zbl 06160396

[Bailesteanu et al. 2010] M. Bailesteanu, X. Cao, and A. Pulemotov, "Gradient estimates for the heat equation under the Ricci flow", J. Funct. Anal. 258:10 (2010), 3517-3542. MR 2011b:53153 Zbl 1193.53139

[Bao 2007] D. Bao, "On two curvature-driven problems in Riemann-Finsler geometry", pp. 19-71 in Finsler geometry: in memory of Makoto Matsumoto (Sapporo, 2005), edited by S. V. Sabau and H. Shimada, Adv. Stud. Pure Math. 48, Math. Soc. Japan, Tokyo, 2007. MR 2009d:53102 Zbl 1147.53018

[Bao and Robles 2004] D. Bao and C. Robles, "Ricci and flag curvatures in Finsler geometry", pp. 197-259 in A sampler of Riemann-Finsler geometry, edited by D. Bao et al., Math. Sci. Res. Inst. Publ. 50, Cambridge University Press, 2004. MR 2005k:53124 Zbl 1076.53093

[Bidabad and Yarahmadi 2014] B. Bidabad and M. Yarahmadi, "On quasi-Einstein Finsler spaces", Bull. Iranian Math. Soc. 40:4 (2014), 921-930. MR 3255407

[Cao 2008] X. Cao, "Differential Harnack estimates for backward heat equations with potentials under the Ricci flow", J. Funct. Anal. 255:4 (2008), 1024-1038. MR 2009e:35121 Zbl 1146.58014

[Cao and Hamilton 2009] X. Cao and R. S. Hamilton, "Differential Harnack estimates for timedependent heat equations with potentials", Geom. Func. Anal. 19 (2009), 989-1000. MR 2010j:53124 Zbl 1183.53059

[Cao et al. 2013] X. Cao, B. Fayyazuddin Ljungberg, and B. Liu, "Differential Harnack estimates for a nonlinear heat equation”, J. Funct. Anal. 265:10 (2013), 2312-2330. MR 3091816 Zbl 1285.58009

[Hamilton 1982] R. S. Hamilton, "Three-manifolds with positive Ricci curvature”, J. Differential Geom. 17:2 (1982), 255-306. MR 84a:53050 Zbl 0504.53034 
[Kuang and Zhang 2008] S. Kuang and Q. S. Zhang, "A gradient estimate for all positive solutions of the conjugate heat equation under Ricci flow”, J. Funct. Anal. 255:4 (2008), 1008-1023. MR 2009h:53150 Zbl 1146.58017

[Liu 2009] S. Liu, "Gradient estimates for solutions of the heat equation under Ricci flow", Pacific J. Math. 243:1 (2009), 165-180. MR 2010g:53122 Zbl 1180.58017

[Ohta 2009] S.-I. Ohta, "Finsler interpolation inequalities", Calc. Var. Partial Differential Equations 36:2 (2009), 211-249. MR 2011m:58027 Zbl 1175.49044

[Ohta 2011] S.-I. Ohta, "Vanishing S-curvature of Randers spaces", Differential Geom. Appl. 29:2 (2011), 174-178. MR 2012b:53156 Zbl 1215.53067

[Ohta and Sturm 2009] S.-I. Ohta and K.-T. Sturm, "Heat flow on Finsler manifolds", Comm. Pure Appl. Math. 62:10 (2009), 1386-1433. MR 2010j:58058 Zbl 1176.58012

[Ohta and Sturm 2014] S.-I. Ohta and K.-T. Sturm, "Bochner-Weitzenböck formula and Li-Yau estimates on Finsler manifolds", Adv. Math. 252 (2014), 429-448. MR 3144236 Zbl 06284328

[Perelman 2002] G. Perelman, "The entropy formula for the Ricci flow and its geometric applications", preprint, 2002. Zbl 1130.53001 arXiv math/0211159

[Shen 2004] Z. Shen, "Landsberg curvature, S-curvature and Riemann curvature", pp. 303-355 in A sampler of Riemann-Finsler geometry, edited by D. Bao et al., Math. Sci. Res. Inst. Publ. 50, Cambridge University Press, 2004. MR 2005k:53132 Zbl 1074.53063

[Sun 2011] J. Sun, "Gradient estimates for positive solutions of the heat equation under geometric flow”, Pacific J. Math. 253:2 (2011), 489-510. MR 2878821 Zbl 1235.53070

[Wu and Xin 2007] B. Y. Wu and Y. L. Xin, "Comparison theorems in Finsler geometry and their applications”, Math. Ann. 337:1 (2007), 177-196. MR 2007h:53121 Zbl 1111.53060

[Xia 2014] C. Xia, "Local gradient estimate for harmonic functions on Finsler manifolds", Calc. Var. Partial Differential Equations 51:3-4 (2014), 849-865. MR 3268873 Zbl 1316.53083

Received May 8, 2014. Revised March 10, 2015.

SAJJAD LAKZIAN

HAUSDORFF CENTER FOR MATHEMATICS

UNIVERSITÄT BONN

VILLA MARIA

ENDENICHER ALLEE 62

D-53115 BONN

GERMANY

lakzians@gmail.com 


\title{
PACIFIC JOURNAL OF MATHEMATICS
}

\author{
msp.org/pjm
}

Founded in 1951 by E. F. Beckenbach (1906-1982) and F. Wolf (1904-1989)

\section{EDITORS}

Don Blasius (Managing Editor)

Department of Mathematics

University of California

Los Angeles, CA 90095-1555

blasius@math.ucla.edu

\author{
Paul Balmer \\ Department of Mathematics \\ University of California \\ Los Angeles, CA 90095-1555 \\ balmer@math.ucla.edu \\ Robert Finn \\ Department of Mathematics \\ Stanford University \\ Stanford, CA 94305-2125 \\ finn@math.stanford.edu \\ Sorin Popa \\ Department of Mathematics \\ University of California \\ Los Angeles, CA 90095-1555 \\ popa@math.ucla.edu
}

\author{
Vyjayanthi Chari \\ Department of Mathematics \\ University of California \\ Riverside, CA 92521-0135 \\ chari@math.ucr.edu \\ Kefeng Liu \\ Department of Mathematics \\ University of California \\ Los Angeles, CA 90095-1555 \\ liu@math.ucla.edu \\ Jie Qing \\ Department of Mathematics \\ University of California \\ Santa Cruz, CA 95064 \\ qing@ cats.ucsc.edu
}

\section{PRODUCTION}

Silvio Levy, Scientific Editor, production@msp.org

\section{SUPPORTING INSTITUTIONS}

ACADEMIA SINICA, TAIPEI

CALIFORNIA INST. OF TECHNOLOGY

INST. DE MATEMÁTICA PURA E APLICADA

KEIO UNIVERSITY

MATH. SCIENCES RESEARCH INSTITUTE

NEW MEXICO STATE UNIV.

OREGON STATE UNIV.

\author{
STANFORD UNIVERSITY \\ UNIV. OF BRITISH COLUMBIA \\ UNIV. OF CALIFORNIA, BERKELEY \\ UNIV. OF CALIFORNIA, DAVIS \\ UNIV. OF CALIFORNIA, LOS ANGELES \\ UNIV. OF CALIFORNIA, RIVERSIDE \\ UNIV. OF CALIFORNIA, SAN DIEGO \\ UNIV. OF CALIF., SANTA BARBARA
}

\author{
Daryl Cooper \\ Department of Mathematics \\ University of California \\ Santa Barbara, CA 93106-3080 \\ cooper@math.ucsb.edu \\ Jiang-Hua Lu \\ Department of Mathematics \\ The University of Hong Kong \\ Pokfulam Rd., Hong Kong \\ jhlu@maths.hku.hk \\ Paul Yang \\ Department of Mathematics \\ Princeton University \\ Princeton NJ 08544-1000 \\ yang@math.princeton.edu
}

These supporting institutions contribute to the cost of publication of this Journal, but they are not owners or publishers and have no responsibility for its contents or policies.

See inside back cover or msp.org/pjm for submission instructions.

The subscription price for 2015 is US \$420/year for the electronic version, and \$570/year for print and electronic.

Subscriptions, requests for back issues and changes of subscribers address should be sent to Pacific Journal of Mathematics, P.O. Box 4163, Berkeley, CA 94704-0163, U.S.A. The Pacific Journal of Mathematics is indexed by Mathematical Reviews, Zentralblatt MATH, PASCAL CNRS Index, Referativnyi Zhurnal, Current Mathematical Publications and Web of Knowledge (Science Citation Index).

The Pacific Journal of Mathematics (ISSN 0030-8730) at the University of California, c/o Department of Mathematics, 798 Evans Hall \#3840, Berkeley, CA 94720-3840, is published twelve times a year. Periodical rate postage paid at Berkeley, CA 94704, and additional mailing offices. POSTMASTER: send address changes to Pacific Journal of Mathematics, P.O. Box 4163, Berkeley, CA 94704-0163.

PJM peer review and production are managed by EditFLOW ${ }^{\circledR}$ from Mathematical Sciences Publishers.

\section{PUBLISHED BY}

\section{mathematical sciences publishers \\ nonprofit scientific publishing}

http://msp.org/

(C) 2015 Mathematical Sciences Publishers 


\section{PACIFIC JOURNAL OF MATHEMATICS}

Volume $278 \quad$ No. $2 \quad$ December 2015

Differential Harnack and logarithmic Sobolev inequalities along

Ricci-harmonic map flow

ABIMBOLA ABOLARINWA

On $J$-holomorphic curves in almost complex manifolds with

291

asymptotically cylindrical ends

ERKAO BAO

Integration of coupling Dirac structures

OLIVIER BRAHIC and RUI LOJA FERNANDES

Asymptotic behavior of Palais-Smale sequences associated with fractional Yamabe-type equations

Yi FANG and MARÍA DEL MAR GONZÁLEZ

$K$-theory and homotopies of 2-cocycles on higher-rank graphs

ELIZABETH GILLASPY

Fusion products and toroidal algebras

DENIZ KUS and PETER LiTTELMANN

Differential Harnack estimates for positive solutions to heat equation under Finsler-Ricci flow

\section{SAJJAD LAKZIAN}

On the one-endedness of graphs of groups

NichOLAS TOUIKAN

On the structure of vertex cuts separating the ends of a graph

GARETH R. WILKES 